\title{
A Female Patient With Clinical Symptoms as Recurrent Urinary Tract Infection Caused by Urinary Tract Tuberculosis
}

\author{
Rastita Widyasari $^{1}$, Artaria Tjempakasari ${ }^{2 *}$, Chandra Irwanadi Mohani ${ }^{2}$ \\ ${ }^{1}$ Department of Internal Medicine, Faculty of Medicine, Universitas Airlangga - Dr. Soetomo General Hospital Surabaya, \\ Indonesia. \\ ${ }^{2}$ Division of Nephrology and Hypertension, Department of Internal Medicine, Faculty of Medicine, Universitas Airlangga - \\ Dr. Soetomo General Hospital Surabaya, Indonesia.
}

\begin{abstract}
A B S T R A C T
Background: Urinary tract tuberculosis (TB) is one type of extrapulmonary TB. The prevalence in developed countries is around $15-20 \%$ of all cases of extrapulmonary TB. The insidious onset and non-specific constitutional symptoms of urinary tuberculosis often lead to delayed diagnosis and rapid progression to a non-functioning kidney. The only way to limit renal function loss and destruction is by early diagnosis and therapy.

Case: 34 -year-old woman, came with complaints of urinary pain accompanied by right flank pain 10 months prior. Patient also had complaint of weight loss but ignoring complaints of night sweats. Patient repeatedly diagnosed as a urinary tract infection and received many kinds of antibiotic therapy but her complaints were not getting better. Urine production was about $1700 \mathrm{cc} / 24$ hours. From general physical examination, there was a lack of nutritional status with BMI $17.1 \mathrm{~kg} / \mathrm{m} 2$. Vesicular lung sound without rhonchi heard in both lung fields. From the urinalysis examination there were pyuria and haematuria without bacteriuri. Laboratory examination showed value of BUN was $17 \mathrm{mg} / \mathrm{dl}$ and creatinine $0.9 \mathrm{mg} / \mathrm{dl}$. From aerob urine culture we found sterile urine. But we found positive result of Mycobacterium tuberculosis (MTB) urine cultures which was sensitive to isoniazid, rifampicin, pyrazinamide, and ethambutol. Abdominal ultrasound showed severe ecstasis of right pelviocalyceal system without stones,mass, nor cyst. We had additional data from intravenous pyelogram (IVP) which showed a nonvisualized dextra pelviocalyceal system and delayed bladder emptying function at 120th minutes. From computed tomography stonographic, we found severe right hydronephrosis, proximal to distal right hydroureter, and thickening of bladder wall $( \pm 1.61 \mathrm{~cm})$ on the right anterolateral side. To find out the cause of thickening of bladder wall, we did bladder biopsy which showed the mononuclear inflammatory cell stroma. Patients were diagnosed with urinary tract TB and received category 1 of oral anti tuberculosis therapy (Rifampicin, Isoniazid, Pyrazinamid, and Ethambutol) for 12 months and underwent right DJ stent implantation to manage the ectasys.

Conclusion : Urinary tract TB often showed unspecified complaints and can be suggested as recurrent urinary tract infections. Early diagnosis and optimal management were needed to prevent anatomical and functional complications.
\end{abstract}

Correspondence: Artaria Tjempakasari

E-mail: artnef@yahoo.com

Article history: • Received 30 December $2019 \bullet$ Received in revised form 09 January $2020 \bullet$ Accepted 27 January 2020

\section{INTRODUCTION}

Urinary tract infection (UTI) is an inflammatory response of the urothelium to bacterial invasion that is usually associated with bacteriuria and pyuria. In while, a recurrent UTI is infection of urinary tract that occurs after documented with successful resolution of an antecedent infection (Schaeffer et al., 2016). Chronic nonspecific urinary infections may be confused with genitourinary tuberculosis (GUTB). Absence of response to usual antibiotics should raise suspicion of urinary TB (Figueiredo and Lucon, 2008, Visweswaran and Suresh., 2000).

Tuberculosis (TB) is a major global health problem. According to a recent report by the World Health Organization (WHO), there were almost 8.6 million new cases of active TB worldwide. WHO estimates that one third of the world's population is infected with Mycobacterium tuberculosis in its latent form (WHO,
2013). Extrapulmonary sites account for $10 \%$ of TB cases. The frequency of genitourinary tuberculosis (GUTB) in developed countries approaches $15 \%$ to $20 \%$. GUTB also has been found in about $5 \%$ of active TB cases in the nonHIV-infected population. In developed countries, GUTB is the second most common form of extrapulmonary TB after peripheral lymphadenopathy (Figueiredo and Lucon, 2008, Visweswaran and Suresh., 2000, Daher Ede et al., 2013, Rai et al., 2009).

Symptoms and signs of GUTB are commonly nonspecific. Patients are often treated for other bacterial infections (sometimes repeatedly) or are evaluated for possible malignancy before GUTB is entertained (Figueiredo and Lucon, 2008). GUTB can lead to irreversible tissue damage with serious consequences such as renal failure, making it critical for clinicians to consider $\mathrm{TB}$ in the differential diagnosis of genitourinary disorders. 
TB can mimic many other diseases and complicate the correct diagnosis and treatment of infected patients (Chang et al., 2016).

\section{CASE REPORT}

A female, Mrs. T, 34 y.o, work as factory labor, married, a moslem, live in Krajan, Ngreco, Pacitan came to Nephrology Outpatient Clinic Dr.Soetomo General Hospital with chief complaint of pain during urination. Patient was referred from Pacitan public hospital with diagnosis of recurrent urinary tract infection + chronic cystitis.

Patient felt pain during urination since 10 months prior coming to Dr.Soetomo General Hospital Outpatient Clinic eventough received many kind of medical therapies. Pain during urination was described as excessive sensation to urinate and felt discomfort during urinate. Urine flew slowly and ended with dissatisfied sensation. There were no complaints of leucorrhea, cloudy urine, pelvic pain, and changes in menstrual cycle. Patient did not complaining fever nor appetite decreasing. Nevertheless, patient complained weight loss of $46 \mathrm{kgs}$ to $41 \mathrm{kgs}(5 \mathrm{kgs})$ in 10 months. Her urine output was about $1700 \mathrm{cc} / 24$ hours. There were no history of bladder stones, trauma, tumour, or previous cathether using. There were no history of diabetes, chronic cough, and anti-tuberculosis drugs. There were no history of free-sex habitual, tattoo, nor drugs consumption. Her husband did not feel the same complaint. Patient have 2 children and there was no history of miscarriage. From family history taking, there were no history of similar complaints, tumour, nor chronic cough.

From medical history, patient was diagnosed as urinary tract infection by general practitioners in Pacitan 10 months ago and got antibiotic. Patient felt better after treatment, but the same complaint occured again 2 weeks later. Patient was given another kind of antibiotic, but a month later, patient felt the same complaint in more severe pain than before and accompanied with right back pain and pinkish-bloody urine sometimes. Patient decided to have consultation to general surgeon. She was diagnosed as appendicitislikely and underwent surgical removal of the appendix. However, after 3 months post-surgery evaluation, the patient did not get better condition. So that,patient decided to get consultation to internist at Madiun general hospital and was advised to do laboratory examination of blood and urine, abdominal X-rays, and abdominal ultrasound. Patient had been told that she had swelling of the right kidney revealed mild stages without stones found in the urinary tract. Patient had been advised to get consultation to an urologist and did some examination of IVP, cystoscopy, even bladder tissue biopsy. Patient had been diagnosed as chronic bladder infections and received antibiotic and antiinflammatory agent. However, the complaints still did not improve, so that she was referred to Nephrology Outpatient Clinic Dr.Soetomo General Hospital.

When patient came to Nephrology Outpatient Clinic Dr.Soetomo General Hospital, patient was in fine general condition, blood pressure 120/70 $\mathrm{mmHg}$, axillary temperature $37,5^{\circ} \mathrm{C}$. Her BMI was $17.1 \mathrm{kgs} / \mathrm{m}^{2}$. From head and neck examination, there weren't anemia nor dyspnea. There weren't enlarged of lymph nodes. From lung examination, there were vesicular sound throughout the lung fields without additional sound as ronchi nor wheezing. From abdominal examination, there was right flank pain with positive sign of right costovertebral pain.

Patient brought laboratory results from Madiun public hospital (4 month prior) which showed normal blood counts, leucocyturia +4 , erythrocyturia +2 , and negative result of proteinuria, nitrit, glucose, bacteria, and ketone from urinalysis. The value of BUN was $11 \mathrm{mg} / \mathrm{dL}$, serum creatinine $0,82 \mathrm{mg} / \mathrm{dL}$, uric acid $3,8 \mathrm{mg} / \mathrm{dl}$, albumin $4,5 \mathrm{~g} /$ dL, blood glucose $88 \mathrm{mg} / \mathrm{dL}$, AST $27 \mathrm{U} / \mathrm{L}$, ALT $27 \mathrm{u} / \mathrm{L}$, potassium $4.5 \mathrm{mmol} / \mathrm{l}$, sodium $135 \mathrm{mmol} / \mathrm{l}$, chloride 98 mmol/l, and HbsAg negative. Chest X-ray examination showed no fibroinfiltrate nor any abnormalities of lung parenchyme. KUB examination showed normal imaging, no radio-opaque shadow stone had showed. Abdominal ultrasound examination showed mild ectasys of right kidney, but no stone found in the urinary tract. Left kidney and another organs include uterus and adnexa within normal limit. IVP examination showed non-visualized ren dextra pelviocalyceal system because of unclear causes. Patient also brought result of bladder biopsy that mentioned a sectional tissue coated by transitional epithelium and a lot of mononuclear inflammatory cells with no signs of malignancy and concluded as chronic inflammation.

From all data we had been received, we diagnosed patient as suspect recurrent urinary tract infection, right hydronephrosis, and chronic cystitis. Patient was planned to re-examine urinalysis and urine sediment, blood count, LED, RFT, urine culture, and abdominal ultrasonography. Patient was also planned to had consultation with obstetrics and gynecology department to rule out the possibility of gynecology infection or another gynecology abnormality.

The result of urinalysis were leucocyturia +4 , erythrocyturia +2 , negative result of nitrite, glucose, and proteinuria. Urine sediment obtained leukocytes 50100 cells/field, erythrocyte $25-50$ cells/field, isomorphic erythrocytes $(+)$, and there were not bacteria and erythrocyte cast. Laboratory results obtained $\mathrm{Hb} 13.5 \mathrm{~g} / \mathrm{dl}$, WBC 5.600 $\mathrm{g} / \mathrm{dl}$, neutrophils $57 \%$, lymphocytes $21 \%$, platelets 338,000 $/ \mathrm{uL}$, LED $49 \mathrm{~mm} / \mathrm{h}$, BUN $17 \mathrm{mg} / \mathrm{dl}$, serum creatinine 0.9 $\mathrm{mg} / \mathrm{dl}$, potassium $4.2 \mathrm{mmol} / 1$, sodium $135 \mathrm{mmol} / 1$, chlor $100 \mathrm{mmol} / \mathrm{l}$. Urine culture showed no bacteria. Chest X-ray showed normal lung fields (picture 1). Abdominal ultrasonography showed severe right hydronephrosis with no stones appeared along urinary tract (picture 2). Consultation to gynecology department had been answered and gynecological status of patient was normal, there were no pelvic infections, endometriosis, nor malignancy. From all data we received, we advised patient to undergo CTstonografi to find any possibility of urinary tract stones that lead to hydronephrosis. The result of of CT-stonography (picture 3) showed that there was severe ectasys in pyelocalyceal system in right kidney with dilatation of right ureter from proximal to distal.There were no calcification nor stones along urinary tract. Left kidney within normal limit. In bladder, there was a thickening of bladder wall $( \pm$ $1.61 \mathrm{~cm}$ ) on the antero-lateral of the right side.

We decided to undergo urine cytologic examination, review of biopsy specimen reading, urine culture of Mycobacterium tuberculosis (MTB), and PCR-TB. 
However, patient refused to examine PCR-TB of urine because of its cost. Patient was also consulted to the urology department for management of dextra severe hydronephrosis and hydroureter. The result of urine cytology showed no malignant cells in the specimen. The review of biopsy specimen reading showed the same conclusion which was chronic inflammation with a lot of mononuclear cells. From consultation to urologist, patient was advised to undergo re-examination of IVP. Results of IVP (picture 4A-4B) : delayed of right kidney function up to minute $120^{\text {th }}$. With normal functions of left kidney and left ureter. Then, patient was advised to undergo an RPG (+ URS dextra if necessary) and dextra DJ-stent mounting after result of MTB urine culture was accepted. After 2 months of waiting, we received the result of of urine MTB culture that obtained there was a growing of Mycobacterium tuberculosis which were sensitive to all of $1^{\text {st }}$ category of anti-tuberculosis drugs (rifampicin, isoniazid, pyrazinamide, and ethambutol).

From all data, we diagnosed patient as urinary tract tuberculosis with complication of severe right hydronephrosis and hydroureter ec thickening of bladder wall. Patient had been planned to receive $1^{\text {st }}$ category of anti-tuberculosis drug regiment for 12 months. and undergo consultation to urologist for surgical procedure. The pretherapy laboratory examination showed value of BUN was $13 \mathrm{mg} / \mathrm{dl}$, creatinin serum $0.65 \mathrm{mg} / \mathrm{dl}$, creatinin clearance of 24-hour urine production $93,1 \mathrm{ml} / \mathrm{min} / 1,73 \mathrm{~m}^{2}$, AST 18 $\mathrm{U} / \mathrm{L}, \mathrm{ALT} 24 \mathrm{U} / \mathrm{L}$, and negative result of HIV rapid test. The body weight of patient was $41 \mathrm{kgs}$. We advised patient to take 3 tablets of 4-Fixed Drugs Combination $\left(\mathrm{H}_{75} \mathrm{R}_{150}\right.$ $Z_{400} E_{275}$ ) everyday in 2-month period of initial intensive phase and followed with 3 tablets of 2-Fixed Drugs Combination $\left(\mathrm{H}_{150} \mathrm{R}_{150}\right) 3$ times a week for 10 months of continuation phase. Patient was also advised by urologist to undergo surgical treatment (RPG dextra + dextra DJstent mounting) to manage right severe hydronephrosis and hydroureter, but patient refused .

After completing anti-tuberculosis therapy program in 12 months, patients's body weight was increasing from $42 \mathrm{kgs}$ to $46 \mathrm{kgs}$. Patient was also advised to undergo re-evaluation of urynalisis, urine culture of MTB, and abdominal ultrasound. Re-evaluation of urinalysis showed erytrocyturia +1 without leucocyturia. Re-evaluation of urine culture showed no growth of Mycobacterium tuberculosis. Re-evaluation of abdominal ultrasound (picture 5) showed that was still seemed severe ectasys of dextra pelviocalyceal system, no stone nor mass in urinary tract.

\section{DISCUSSION}

Extrapulmonary sites account for $10 \%$ of all TB cases. The genitourinary tract is a primary target of hematogenous infection of Mycobacterium tuberculosis. In developed countries, genitourinary tuberculosis (GUTB) is the second most common form of extrapulmonary TB after peripheral lymphadenopathy. The frequency in developed countries approaches $15 \%$ to $20 \%$ of all TB cases. GUTB occurs in $5 \%$ of active TB cases in non-HIV-infected population (Figueiredo and Lucon, 2008, Visweswaran and Suresh., 2000, Rai et al., 2009, Daher Ede et al., 2013). The kidney is the most common site of GUTB. GUTB usually affects adults between the second and fourth decades of life because there is a long latent period (5-40 years) between the original pulmonary infection and the appearance of clinical renal disease (Merchant et al., 2013).

Urinary tract TB may be asymptomatic, nonspecific, or may mimic other disorders. Patients may present with constitutional symptoms or symptoms related to the lower urinary tract and often treated for other bacterial infections (sometimes repeatedly) or are evaluated for possible malignancy. Urinary tract TB can be progressive and destructive but symptomatically silent until it extends into the bladder (Figueiredo and Lucon, 2008). The urinary tract TB has varied presentation and some of the common ways are recurrent or resistant urinary tract infection, sterile pyuria with or without haematuria, irritative voiding symptoms, hydronephrosis, infertility and pelvic inflammatory disease, or renal failure. The most common symptoms are the forms irritative voiding (frequency, urgency, dysuria), which are found in more than $50 \%$ of the patients. Frequency and urgency related to reduced bladder capacity. Incomplete emptying, increased susceptibility to infection, and secondary vesicoureteral reflux (VUR) may also occur. In chronic ureteral obstruction, an enlarged kidney leads to a pain. Many a times, the patient is asymptomatic, but $90 \%$ of all cases show chronic sterile pyuria. Gross haematuria is seen in only $10 \%$, but microscopic haematuria is present in up to $50 \%$ of the cases. Macroscopic haematuria in urinary TB is a result of bleeding from the ulcerating lesions, inflammation of the urothelium, or rupture of a blood vessel in the vicinity of a cavity (Visweswaran and Suresh., 2000, Kapoor et al., 2008). The typical TB constitutional symptoms of fever, weight loss, night sweats, and malaise are present in fewer than $20 \%$ of patients. Up to $50 \%$ of patients with urinary tract TB have only dysuria on presentation. End-stage renal failure develops in approximately $7 \%$ of patients (Figueiredo and Lucon, 2008, Visweswaran and Suresh., 2000).

The current gold standard for the diagnosis of urinary tract TB is urine acid-fast bacilli (AFB) culture. First-void urine is the best sample because urine is the most concentrated at that time. These should be cultured immediately after collection because prolonged exposure to urine acidity can retard mycobacterial growth (Society, 2000, Kulchavenya, 2014). Another tests are amplification tests (PCR) and tuberculin skin test (TST) which have been developed to speed the detection of MTB infection, providing results within 1 to 2 days but they have limited usefulness in the diagnosis of active disease (Visweswaran and Suresh., 2000). Imaging examinations as abdominal USG, intravenous urography, MRI, CT scan, cystoscopy and ureteroscopy may need to find the anatomical complication of urinary tract TB (Merchant et al., 2013, Chang et al., 2016). Cystoscopy or ureteroscopy plays a limited role in the diagnosis of TB. Although it allows direct visualization of lesions, findings can be nonspecific. They include local hyperemia, mucosal erosion, ulceration, granulomatous masses, and irregularity of the ureteral orifices. Biopsies should be performed when possible. Bladder biopsy can be $19 \%$ to $52 \%$ sensitive for TB (Figueiredo and Lucon, 2008). 
Descending infection to the bladder usually begins near the ureteral orifices and spreads along the lymphatics to other areas. The dome of the bladder is the most affected, whereas the trigone and neck usually remain normal. Mucosal inflammation and haematuria follow this condition (Wong et al., 2013). After approximately a year of chronic inflammation and mucosal scarring, contracted trabeculated bladder with intramural thickening develops. At least $8.9 \%$ of all urinary tract $\mathrm{TB}$ cases have fibrosis of the bladder wall, with capacity reduction and increased frequency of micturition. Symptoms as frequency, urgency, pain, and dysuria become prominent when bladder capacity shrinks to less than $100 \mathrm{~mL}$. Bladder contraction is a late complication of urinary tract TB (Figueiredo and Lucon, 2008, Ronald AR and LE, 2007a). It is also important to identify patients subject to the second major cause of renal functional loss with hydronephrosis secondary to obstruction induced by the tuberculous process (Ronald AR and LE, 2007b).

Urinary tract TB can be treated with the standard shortcourse regimen of 6 months of first-line anti-tuberculosis drugs. Treatment begins with an initial intensive phase of 2 months of daily isoniazid, rifampin, pyrazinamide, and ethambutol then followed by 4 months continuation phase of isonoazid and rifampicin given daily, or alternatively twice weekly. In order to increase patient compliance, antituberculosis drugs can be given as fixed drugs combination (FDC) (MinistryofHealth., 2006). The initial intensive phase of treatment targets rapidly multiplying bacteria, whereas the continuation phase attempts to eradicate slow, sporadic multipliers and persistent bacteria. Although 6 months is the duration of standard short-course therapy, clinical scenarios regularly arise that require prolongation of treatment. Clinical disease present affect duration of treatment (CDC., 2003). Some clinicians recommend 12 months of therapy for urinary tract TB because of high relapse rates of up to $22 \%$ when therapy is given for only 6 months (Chang et al., 2016).

About $55 \%$ of patients with urinary tract $\mathrm{TB}$ will require surgical management during the course of their disease (Wong, et al., 2013). Intervention is more frequent as disease advances. Surgical procedures are performed to relieve urinary obstruction and drain infected material, to remove non-working infected kidneys in cases resisting cure, to improve medically resistant hypertension secondary to a functionally excluded kidney, or to reconstruct the urinary tract. Currently, more than half of operations performed for $\mathrm{TB}$ are reconstructive. The optimal timing of surgery is 4-6 weeks after the initiation of therapy. This delay allows active inflammation to subside, the bacillary load to decrease, and lesions to stabilize (Gupta NP et al., 2008). Ureteral reimplantation, endoscopic balloon dilatation, or implantation of ureteral stents may be necessary if progressive obstruction develops. Nephrectomy is rarely required but may be indicated for intractable pain, untreatable infection proximal to a stricture, uncontrollable hematuria or hypertension, or drug resistance. Bladder augmentation surgery may be required if the bladder is scarred and contracted after tuberculosis infection (Wong et al., 2013).

Response to treatment can be monitored through clinical observation. Patient's body weight is an useful indicator. After 2 months of initial intensive therapy, urine is cultured for $M$. tuberculosis. Patients also should be evaluated of MTB urine culture in 6 and 12 months (Visweswaran and Suresh., 2000, Rai et al., 2009). The prognosis of genitourinary TB depends on the host resistance, the load, and virulence of the organism. Most manifestations result from the complications, which can be prevented by timely therapy of tuberculosis and appropriate surgical intervention when indicated. With the advent of effective therapeutic measures, the long-term complications and sequelae of TB have decreased significantly (Visweswaran and Suresh., 2000).

\section{CONCLUSION}

A female patient, 34 y.o, came to nephrology outpatient clinic of Dr. Soetomo hospital with chief complaint of pain during urination since 10 months prior although she had received any medical and surgery treatments. From anamnesis, physical and additional examination, patient was diagnosed as urinary tract tuberculosis with severe unilateral hydronephrosis and hydroureter ec thickening of anterolateral of right side of bladder wall as its complication. Patient received 12 months of first-line anti-tuberculosis drugs and had been undergo implantation of DJ stent dextra for dextra obstructive uropathy. Patient felt better condition and had weight gaining after completing anti-tuberculosis therapy program. Overall prognosis of patient was good.

\section{CONFLICT OF INTEREST}

The author declare there is no conflict of interest of this study.

\section{REFERENCES}

CDC. 2003. Treatment Of Tuberculosis, American Thoracic Society, Centers For Disease Control And Prevention (CDC), And Infectious Diseases Society Of America. .

Chang, A., Blackburn, B. \& Hsieh, M. 2016. Tuberculosis And Parasitic Infections Of The Genitourinary Tract.

Daher Ede, F., Da Silva, G. B., Jr. \& Barros, E. J. 2013. Renal Tuberculosis In The Modern Era. Am J Trop Med Hyg, 88, 54-64.

Figueiredo, A. A. \& Lucon, A. M. 2008. Urogenital Tuberculosis: Update And Review Of 8961 Cases From The World Literature. Reviews In Urology, 10, 207-217.

Gupta Np, Kumar A \& S., S. 2008. Reconstructive Bladder Surgery In Genitourinary Tuberculosis. Indian J Urol 2008;24(3):382-7.

Kapoor, R., Ansari, M. S., Mandhani, A. \& Gulia, A. 2008. Clinical Presentation And Diagnostic Approach In Cases Of Genitourinary Tuberculosis. Indian Journal Of Urology : Iju : Journal Of The Urological Society Of India, 24, 401-405.

Kulchavenya, E. 2014. Urogenital Tuberculosis: Definition And Classification. Therapeutic Advances In Infectious Disease, 2, 117-122.

Merchant, S., Bharati, A. \& Merchant, N. 2013. Tuberculosis Of 
The Genitourinary System-Urinary Tract Tuberculosis: Renal Tuberculosis-Part Ii. The Indian Journal Of Radiology \& Imaging, 23, 64-77.

Ministryofhealth. 2006. Obat Anti Tuberkulosis. Pedoman Nasional Penanggulangan Tuberkulosis Ed 2. Jakarta Indonesian Ministry Of Health.

Rai, A., Pahwa H, Jain V \& S., M. 2009. Management Of Genito-Urinary Tuberculosis. The Internet Journal Of Surgery Volume 23

Ronald Ar \& Le, N. 2007a. Infections Of The Upper Urinary Tract.

Ronald Ar \& Le, N. 2007b. Infections Of The Upper Urinary Tract.
Schaeffer, A., Matulewicz, R. \& Klumpp, D. 2016. Infection Of Urinary Tract.

Society, A. T. 2000. Diagnostic Standards And Classification Of Tuberculosis In Adults And Children. Am J Respir Crit Care Med, 161, 1376-95.

Visweswaran, R. \& Suresh., B. 2000. Tuberculosis Of The Urinary Tract.

WHO. 2013. Tuberculosis Fact Sheet No. 104.

Wong, N., Hoag, N. A., Jones, E. C., Rowley, A., Mcloughlin, M. G. \& Paterson, R. F. 2013. Genitourinary Tuberculosis Masquerading As A Ureteral Calculus. Canadian Urological Association Journal = Journal De L'association Des Urologues Du Canada, 7, E363-E366. 